\title{
Retraction Note to: A SERS-based lateral flow assay for the stroke biomarker S100- $\beta$
}

\author{
Ying Wang ${ }^{1} \cdot$ Yajun Hou $^{1} \cdot{\text { Hanxia } \mathrm{Li}^{1} \cdot \text { Mingfeng Yang }}^{1} \cdot$ Peng Zhao ${ }^{2} \cdot$ Baoliang Sun $^{1}$ \\ (C) Springer-Verlag GmbH Austria, part of Springer Nature 2021
}

\section{Retraction Note to: Microchimica Acta (2019) 186: 548 https://doi.org/10.1007/s00604-019-3634-z}

The Editor-in-Chief has retracted this article [1] because some of the figures appear to overlap with figures in previously published articles, specifically:

- The Raman mapping data in Figure 3a appear to overlap with the Raman mapping data in Figure 4a of Zhang et al. [2]

- The top right image in Figure 5a appears to be the same as the left panel of Figure S4 of Fu et al. [3]

The online version of the original article can be found at https://doi.org/ 10.1007/s00604-019-3634-z

Peng Zhao

pengz9@163.com

$\triangle$ Baoliang Sun

blsun88@163.com

1 Key Lab of Cerebral Microcirculation in Universities of Shandong, Shandong First Medical University \& Shandong Academy of Medical Sciences, Taian 271000, Shandong, China

2 Radiology College, Shandong First Medical University \& Shandong, Academy of Medical Sciences, Taian 271000, Shandong, China
The Editor-in-Chief therefore considers the data reported in this article to be unreliable. All authors agree with this retraction.

\section{Compliance with ethical standards}

Conflict of interest The authors declare that they have no competing of interests.

\section{References}

1. Wang, Y., Hou, Y., Li, H. et al. A SERS-based lateral flow assay for the stroke biomarker S100- $\beta$. Microchim Acta (2019) 186: 548. https://doi.org/10.1007/s00604-019-3634-z.

2. Di Zhang, Li Huang, Bing Liu, Haibin Ni, Liangdong Sun, Enben $\mathrm{Su}$, Hongyuan Chen, Zhongze Gu, Xiangwei Zhao, Quantitative and ultrasensitive detection of multiplex cardiac biomarkers in lateral flow assay with core-shell SERS nanotags. Biosensors and Bioelectronics, Volume 106, 2018, Pages 204-211. https://doi.org/ 10.1016/j.bios.2018.01.062.

3. Xiuli Fu, Ziyi Cheng, Jimin Yu, Priscilla Choo, Lingxin Chen, Jaebum Choo, A SERS-based lateral flow assay biosensor for highly sensitive detection of HIV-1 DNA, Biosensors and Bioelectronics, Volume 78, 2016, Pages 530-537. https://doi.org/10.1016/j.bios. 2015.11.099.

Publisher's note Springer Nature remains neutral with regard to jurisdictional claims in published maps and institutional affiliations. 